\title{
L-DOPA-elicited abnormal involuntary movements in the rats damaged severely in substantia nigra by 6-hydroxydopamine
}

\author{
Rongfei Wang', Ming Shao ${ }^{2}$ \\ ${ }^{1}$ Department of Neurology, Guangdong Province Hospital of Traditional Chinese Medical, Guangzhou 510120, China; ${ }^{2}$ Department of Neurology, \\ Affiliated Brain Hospital of Guangzhou Medical University, Guangzhou 510370, China \\ Contributions: (I) Conception and design: R Wang, M Shao; (II) Administrative support: None; (III) Provision of study materials or patients: None; (IV) \\ Collection and assembly of data: R Wang; (V) Data analysis and interpretation: R Wang, M Shao; (VI) Manuscript writing: All authors; (VII) Final \\ approval of manuscript: All authors. \\ Correspondence to: Prof. Ming Shao. Department of Neurology, Affiliated Brain Hospital of Guangzhou Medical University, 36 Mingxin Road, Liwan \\ District, Guangzhou 510370, China. Email: yimshao@gzhmu.edu.cn.
}

Background: Dyskinesia of rat models can occur in several conditions: acute levodopa (L-DOPA) administration provided that the drug dose is sufficiently high and/or that the nigrostriatal dopamine (DA) pathway is seriously damaged, and repeated L-DOPA administration which could cause a reduction of the dyskinesia-threshold dose, a progressive aggravation and an increasing incidence of dyskinesia. Therefore, if the damage of the nigrostriatal DA pathway is extremely severe, what abnormal movements can be elicited by first injecting L-DOPA or other dopaminergic agonists? The problem deserves exploring.

Methods: Rat models with damage of varying severity were divided into three groups: the serious lesion [induced by $40 \mu \mathrm{g}$ 6-hydroxydopamine (6-OHDA), two injected coordinates including substantia nigra (SN) and medial forebrain bundle], the moderate lesion $(20 \mu \mathrm{g}$ 6-OHDA, a coordinate in SN) and the control. Three weeks after lesion, the Rota Rad test and Cylinder test were performed to assess the motor activities of rat models, the abnormal involuntary movements (AIMs) elicited by L-DOPA or apomorphine (APO) were observed, and the dopaminergic degeneration in SN and striatum was determined.

Results: Both seriously lesioned rats and the moderately were observed to exhibit a significant decrease in motor activities. In the rats with a serious lesion, scarcely any dopaminergic neurons were present in the SN, tissue DA level decreased by $99 \%$ in the striatum, and both L-DOPA and APO could elicit AIMs and rotational movements. In the rats with the moderate lesion, only rotation movements could be elicited. The rotation speed of moderately lesioned rats was 9 turns/min, but that of seriously was only 4.5 turns/min elicited by APO.

Conclusions: Both dyskinesia and rotation movement are the specific expressions elicited by L-DOPA or APO in rats whose SN is damaged by 6-OHDA. Dyskinesia reflects more severe damage than rotation movement.

Keywords: Parkinson's disease (PD); dyskinesia; abnormal involuntary movements (AIMs); levodopa (L-DOPA); 6-hydroxydopamine (6-OHDA)

Submitted Feb 12, 2020. Accepted for publication Mar 09, 2020.

doi: $10.21037 /$ apm.2020.03.32

View this article at: http://dx.doi.org/10.21037/apm.2020.03.32 


\section{Introduction}

Levodopa (L-DOPA) remains the most efficacious drug for the treatment of Parkinson's disease (PD) but causes adverse effects, such as L-DOPA-induced abnormal involuntary movements (AIMs), which are referred to as L-DOPA-induced dyskinesia (LID) (1). A review shows that approximately $30 \%$ of $\mathrm{PD}$ patients occur dyskinesia after 4-6 years of L-DOPA treatment, and close to $90 \%$ of the patients suffer from this complication after 9 years (2). Rat models of LID are produced by first injuring the nigrostriatal DA pathway by 6-hydroxydopamine (6-OHDA), and then administering L-DOPA once or twice a day for several weeks, mimicking the process of developing dyskinesia in PD patients (3-6).

However, dyskinesia of rat models can occur in several conditions: acute L-DOPA administration provided that the drug dose is sufficiently high and/or that the nigrostriatal DA pathway is seriously damaged, and repeated L-DOPA administration which could result in a reduction of the dyskinesia-threshold dose, a progressive aggravation and an increasing incidence of dyskinesia (7). So, if the damage of the nigrostriatal DA pathway is extremely serious, what abnormal movements can be elicited by first injecting L-DOPA or apomorphine (APO)? The problem deserves to explore.

In the present study, the rats of extremely serious damage in the nigrostriatal DA pathway induced by 6-OHDA have been compared to that of moderate damage concerning their motor abilities and dopaminergic degeneration in $\mathrm{SN}$ and striatum.

\section{Methods}

\section{Experimental animals and groups}

Twenty-seven female Sprague-Dawley rats (190-210 g body weight) used in the experiment were obtained from the Laboratory Animal Center of Guangdong province, China. The rats were housed five per cage on a $12 \mathrm{~h}$ light/ dark cycle and ad libitum access to rodent chow and water. All experimental protocols involving the animals were reviewed and approved by the Ethical Committee of the Guangzhou Medical University. All procedures were carried out following the approved guidelines and regulations of the National Institutes of Health for the care and use of laboratory animals. All the animals were evaluated carefully to make sure none of them have automatic spinning behavior, or sighting impairment or limb motion impairment. The rats were randomly divided into three groups (serious lesion, moderate lesion, and control), and nine rats were in each group. After the behavioral testing, all the rats were sacrificed, then six animals were randomly selected for immunohistochemical detection in each group, and three for determining the DA level in the striatum.

\section{Establishment of the model}

The rats were anesthetized with $1 \%$ Pentobarbital $(4 \mathrm{~mL} /$ $\mathrm{kg}$, i.p.) and fixed in a stereotaxic device with a horizontal cranium position. The rats in the serious-lesion group were injected unilaterally in the right substantia nigra compacta (SNc) and right medial forebrain bundle (MFB) respectively with $4 \mu \mathrm{L}$ 6-OHDA (Sigma, $5 \mu \mathrm{g} / \mu \mathrm{L}$, in $0.2 \%$ ascorbate saline, made on the same day) at a rate of $1 \mu \mathrm{L} / \mathrm{min}$. Injection coordinate of the MFB, anteroposterior (AP): $-4.4 \mathrm{~mm}$, medial-lateral (ML): $-1.2 \mathrm{~mm}$ from the bregma, and dorsoventral (DV): $7.8 \mathrm{~mm}$ from the dura. Injection coordinate of the SNc, AP: $-5.2 \mathrm{~mm}, \mathrm{ML}:-2.2 \mathrm{~mm}$; and DV: $7.8 \mathrm{~mm}$. The rats in the moderate lesion group were injected only in the SNc with $4 \mu \mathrm{L}$ 6-OHDA. The rats in the control group were injected in the SNc with $4 \mu \mathrm{L}$ vehicle $(0.2 \%$ ascorbate saline).

Twenty-one days after lesion, all of the rats were injected with apomorphine (APO, $0.5 \mathrm{mg} / \mathrm{kg}$, i.p.) for detecting AIMs and rotation movements, and both the Rota Rad test and Cylinder test were performed in the following week. Then all the rats were given ip injections of $6 \mathrm{mg} / \mathrm{kg}$ L-DOPA combined with benserazide $(15 \mathrm{mg} / \mathrm{kg})$ on the 28th day after lesion, $10 \mathrm{mg} / \mathrm{kg}$ L-DOPA combined with benserazide $(15 \mathrm{mg} / \mathrm{kg})$ at 35 th day as well as $15 \mathrm{mg} / \mathrm{kg}$ on 42 nd day. After each i.p. injection, the AIMs, and rotation movements were observed.

\section{AIMs rating}

For quantification, the AIMs elicited by L-DOPA or APO, rats were observed individually every $20 \mathrm{~min}$ for $3 \mathrm{~h}$ following administration, during $1 \mathrm{~min}$ (observations were performed between 10.00 and $16.00 \mathrm{~h}$ ). AIMs were classified based on their topographic distribution into four subtypes (8): locomotive dyskinesia, i.e., increased locomotion with contralateral side bias; axial dystonia, i.e., contralateral twisted posturing of the neck and upper body; orolingual dyskinesia, i.e., stereotyped jaw movements and contralateral tongue protrusion; forelimb dyskinesia, i.e., repetitive rhythmic jerks or dystonic posturing of 
the contralateral forelimb, and/or grabbing movement of the contralateral paw. For each of these four subtypes, each rat was scored on a scale from 0 to 4 (1= occasional; 2 = frequent; $3=$ continuous but interrupted by sensory distraction; $4=$ continuous, severe, not interrupted by sensory distraction). The maximum AIMs score per observation was 16 .

An added score was given to the amplitude of limb and axial dyskinesia (8). The amplitude score was applied as follow: Axial dystonia: 1= lateral deviation of head and neck $<30^{\circ}, 2=$ lateral deviation of head and neck $30-60^{\circ}, 3=$ lateral deviation or torsion of head, neck and upper trunk $60-90^{\circ}, 4=$ torsion of neck and trunk at $>90^{\circ}$ causing the rat to lose balance; Forelimb dyskinesia: $1=$ tiny oscillatory movements of the paw and the distal forelimb around a fixed position, $2=$ movements of low amplitude but causing visible translocation of both distal and proximal limb, $3=$ translocation of the whole limb with visible contraction of shoulder muscles, $4=$ vigorous limb and shoulder movements of maximal amplitude.

\section{Rota Rod test}

The Rota Rod test was used to assess motor coordination and balance (3). The Rota Rod test was performed by placing the rat on a rotating drum and measuring the time each animal was able to support its balance walking on top of the drum. The diameter of the drum was $7 \mathrm{~cm}$, and its material was the organic glass with a striped surface. Animals were pre-trained at 5 turns/min, twice a day, three days before the test. If animals dropped during this process, they at once placed them back to the rotating drum. Before tested, the rats were first placed on the drum rotating at 5 turns/min to adapt for 1 minute, and then the rotating was stopped to let the rats rest for half a minute. When tested, the Rota Rod test started at the speed of 5 turns $/ \mathrm{min}$, which increased 5 turns/min every half a minute until the rats dropped from the rotating drum, or the time was at most 300 seconds. The animals were touched on their tails several times in each session to support a high degree of alertness in the test (1).

\section{Cylinder test}

The cylinder test was used to assess forelimb use (9). For the test, the rat was placed gently in a transparent cylinder (diameter $20 \mathrm{~cm}$ ) and videotaped for 10 minutes. Mirrors were placed behind the cylinder so that behavior could be viewed when the animal turned away from the camera. Exploratory activity for $3 \mathrm{~min}$ was analyzed using a video recorder with slow-motion capabilities. Forelimb use was observed during the first contact against the wall after rearing and during lateral exploration of the wall. Independent use of contralateral and ipsilateral forelimbs, as well as simultaneous use of both forelimbs, were calculated. After landing, the first forelimb contacting the wall with weight support was scored as an independent wall placement for that limb. A simultaneous (both forelimbs) movement was scored if the first limb supported its position, and the other forelimb was placed on the wall. If the animal placed both forelimbs simultaneously on the wall, that was scored as a simultaneous movement. To score again, the rat had to remove both forelimbs from the surface of the cylinder. After the rat removed both forelimbs from the wall, stood with its hind-limbs, and then contacted the wall with the other forelimb, that was scored as an independent wall placement for that limb. When the rat explored the wall laterally, alternating both limbs, the simultaneous movement was scored. If one forelimb was stationary and the other made several movements, it was scored as one simultaneous movement. Forelimb use during landing could not be measured reliably from the videotapes and was not scored.

Each behavior was expressed in terms of (I) percent use of the contralateral forelimb: the number of contralateral limb use plus half the number of simultaneous movement, then divided by the total number of limb use observations (contralateral, ipsilateral and both limb use observations) for wall movements; (II) percent use of the ipsilateral forelimb: the number of ipsilateral limb use plus half the number of simultaneous movement, then divided by the total number of limb use observations for wall movements.

\section{Determination of TH immunoreactivity}

After the final behavior observation, the rats were anesthetized and terminally killed by decapitation. And their brains were fixed transcardially perfusion with $50-100 \mathrm{~mL}$ 0.1 M phosphate-buffered saline (PBS, pH 7.4) followed by $250 \mathrm{~mL}$ buffered $4 \%$ paraformaldehyde (PFA). Brains were removed and post-fixed in PFA for $24 \mathrm{~h}$ followed by immersion in a solution of $30 \%$ sucrose in PBS until they sank. Serial coronal sections were cut at $25 \mu \mathrm{m}$ on a freezing sled microtome. A series of every sixth section was stained immunohistochemically, free-floating for tyrosine hydroxylase (TH, MAB318, Millipore, USA; 1:1,000). All 

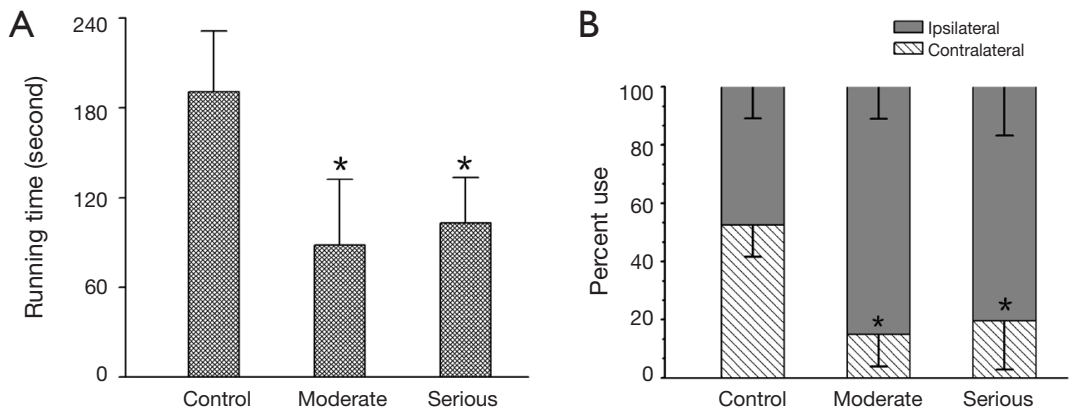

Figure 1 The motor abilities after unilaterally dopaminergic degeneration. The running time on rotating drum in groups of serious lesion, moderate lesion, and control was $103 \pm 30,88 \pm 43$ and $190 \pm 40$ seconds respectively in (A). The percentages of contralateral forelimb contacting wall in groups of serious lesion, moderate lesion and control were 19.6 $\pm 16.8,14.9 \pm 11.0$ and $52.5 \pm 10.9$ respectively in (B). *, $\mathrm{P}<0.01$ serious $v s$. control and moderate $v s$. control (unpaired $t$-test).

immunohistochemical procedures were conducted using the streptavidin-biotin method. Observe the TH positive cells in SNc with an Olympus IX71 microscope.

\section{Determination of $D A$ level in tissue samples of the striatum}

After the rats were anesthetized and killed, their brains were taken out rapidly, removed the dural, flushed the blood, separated the striatum, scaled the wet weight immediately. According to " $0.1 \mathrm{M}$ perchloric acid $(\mu \mathrm{L})$ : the wet weight of striatum $(\mathrm{mg})=50 \mu \mathrm{L}: 10 \mathrm{mg}$ ", the samples were sonicated in cold perchloric acid and centrifuged for $15 \mathrm{~min}$ at $12,000 \mathrm{rpm}$. Then the supernatant was transferred to $0.22-\mu \mathrm{m}$ micropore separators, and the filtrate of the sample was kept frozen $\left(-80^{\circ} \mathrm{C}\right)$ until analysis.

The level of DA in the tissue sample was determined by high-performance liquid chromatography (HPLC) coupled with electrochemical detection. First, the standard sample of DA was prepared. The DA was sonicated in cold $0.1 \mathrm{M}$ perchloric acid and made into 5 graded concentration, i.e., $0.01,0.02,0.05,0.1$, and $0.2 \mu \mathrm{g} / \mathrm{mL}$. Then the standard curve was made, and the DA concentration could be calculated with the standard external method.

Filtrate $(5 \mu \mathrm{L})$ was injected into a reversed-phase C18 column (4 mm Ø, $200 \mathrm{~mm}$ length, Lichrosorb RP). The mobile phase [citric acid $85 \mathrm{mmol} / \mathrm{L}$, sodium citrate $32.8 \mathrm{mmol} / \mathrm{L}, 0.1 \mathrm{mmol} / \mathrm{L}$ EDTA, $3.2 \mathrm{mmol} / \mathrm{L}$ sodium heptane sulfonate and $8 \%$ methanol (v/v), $\mathrm{pH}$ 3.7] was delivered at a rate of $30 \mu \mathrm{L} / \mathrm{min}$. The detection wavelength was UV $280 \mathrm{~nm}$, and the detection voltage of the electrochemical detector was $0.65 \mathrm{~V}$. The detection limit of the assay was $0.6 \mu \mathrm{g} / \mathrm{mL}$ for DA.

\section{Statistical analysis}

All data were analyzed using SPSS for Windows. Integrated AIMs scores and rotational behaviors recorded in different testing sessions were analyzed using repeated-measures ANOVA. Rota Rod performances, Cylinder Test datum, and biochemical test results were analyzed using either paired or unpaired $t$-test where proper. The $\mathrm{P}$-value of less than 0.05 was statistically significant. All data were expressed as group means \pm SEM.

\section{Results}

\section{Motor abilities of the rats suffered 6-OHDA lesion}

Both the seriously lesioned rats and the moderate showed a significant decrease in motor activities. Their running time on the rotating drum was significantly shorter than that of rats with sham-operation, and their use of the impaired forelimb (contralateral to the lesion) was significantly less compared to control (Figure 1).

\section{Assessment of AIMs}

Six weeks after the lesion, both the L-DOPA and APO could elicit many visible motor responses in serious lesion rats. Injecting APO or L-DOPA intraperitoneally for the 
first time could elicit AIMs, which included rhythmic jerks of the forelimb contralateral to the lesion (limb dyskinesia), torsion of axial muscles affecting the neck, trunk and tail (axial dystonia), masticatory movements of the empty mouth with tongue protrusion (orolingual dyskinesia), and controversies turning behavior (locomotive dyskinesia). After injecting $6 \mathrm{mg} / \mathrm{kg}$ L-DOPA, these AIMs peaked at 20 $90 \mathrm{~min}$ and subsided within $150-180 \mathrm{~min}$. When the dose of L-DOPA raised to $10 \mathrm{mg} / \mathrm{kg}$ (7 weeks after lesion) and $15 \mathrm{mg} / \mathrm{kg}$ (8 weeks after lesion), the AIM scores increased, but the duration had minor changes (Figure $2 A, B, C$ ). After injecting $0.5 \mathrm{mg} / \mathrm{kg}$ APO the AIM scores reached a peak at 10-30 min and subsided within 40-60 min (Figure 2A,B,C). The rats with moderate lesion showed no AIMs except for rotational movements after injecting APO.

After injecting $6 \mathrm{mg} / \mathrm{kg} \mathrm{L-DOPA}$, the rats with serious lesion showed rotational movements that peaked at 20-90 min and subsided within 140-160 $\mathrm{min}$. When the dose of L-DOPA raised to $10 \mathrm{mg} / \mathrm{kg}$, even to $15 \mathrm{mg} / \mathrm{kg}$, the rotation speed increased, but the duration had minor changes (Figure 2D). Injecting APO intraperitoneally could also induce rotational movements, the rotation speed reached the peak ( 4.5 turns $/ \mathrm{min}$ ) at $5-30 \mathrm{~min}$, and subsided within 40-60 min (Figure 2D,E). The rats with moderate lesion also exhibited rotational movements after injecting APO, and the rotation speed ( 9 turns/min) was faster significantly than that of the rats with a serious lesion (Figure $2 E, \mathrm{P}<0.05$ ).

\section{Dopaminergic degeneration induced by 6-OHDA}

Rats were sacrificed eight weeks after the lesion. In the rats with a serious lesion, scarcely any dopaminergic neurons were present in the $\mathrm{SN}$ ipsilateral to lesion in $\mathrm{TH}-$ immunostaining. In the rats with a moderate lesion, there was only a $65-76 \%$ reduction. No changes were found in control animals (Figure $3 A, B, C$ ).

In the rats with the serious lesion, the tissue level of DA in striatum contralateral to the lesion was $533.43 \pm 95.26 \mathrm{ng} / \mathrm{mL}$, and that of DA in the ipsilateral side was $5.04 \pm 2.08 \mathrm{ng} / \mathrm{mL}$ (paired $t$-test, $\mathrm{P}<0.01, \mathrm{n}=3$ ) (Figure 3D). In control, the tissue levels of DA were $556.10 \pm 66.58$ and $516.60 \pm 118.37 \mathrm{ng} / \mathrm{mL}$ in the contralateral and ipsilateral side, respectively. For some reason, the tissue level of DA in the rats with moderate lesion did not get acquired.

\section{Discussion}

\section{AIMs occur in rats with serious degeneration of dopaminergic neurons}

6-OHDA first produced the PD rat model in 1968 (10). The 6-OHDA can be injected virtually anywhere along the nigrostriatal tract and induce DA denervation in varying severity (11). The rat models with moderate degeneration of dopaminergic neurons simulate the early and middle stages of $\mathrm{PD}$, and the rat models with serious degeneration mimic the late stage of PD. For rats with moderate degeneration, the injection coordinates include SN, SN territory, and striatum; one or multiple coordinates can be selected, and the total dosage of 6-OHDA is 6-30 $\mu \mathrm{g}(12,13)$. For rats with serious degeneration, the injection coordinates are medial forebrain bundle (MFB) or in combination with $\mathrm{SN}$, and the total dosage of $6-\mathrm{OHDA}$ is $8-32 \mu \mathrm{g}(3-6)$. There is a good correlation between the 6-OHDA dose and lesion severity when the 6-OHDA is injected in the nigrostriatal tract, except for MFB (14). And when injected in MFB, the 6-OHDA can damage serotonin and noradrenergic neurons and induce more severe and rapid lesions (14). Therefore, the MFB has been selected as the sole or one of the injection coordinates for inducing rat models of dyskinesia (3-6).

For extremely serious lesions, we produce the seriously lesioned rats by injecting $40 \mu \mathrm{g}$ 6-OHDA (20 $\mu \mathrm{g}$ in SN and $20 \mu \mathrm{g}$ in MFB), which is higher than that found in the literature. And the rats with moderate lesion are produced by injecting $20 \mu \mathrm{g} 6-\mathrm{OHDA}$ in $\mathrm{SN}$. TH immunohistochemistry shows the different-degree degeneration of dopaminergic neurons between moderately and seriously lesioned rats, and scarcely any dopaminergic neurons in the $\mathrm{SN}$ of serious-lesion rats (Figure 3C). The determination by HPLC shows that in serious-lesion rats, the tissue level of DA reduces about $99 \%$ in the striatum ipsilateral to the lesion side compared with the contralateral intact side (Figure 3D). And the tissue level of dihydroxyphenyl acetic acid (DOPAC, a metabolite of DA) also 

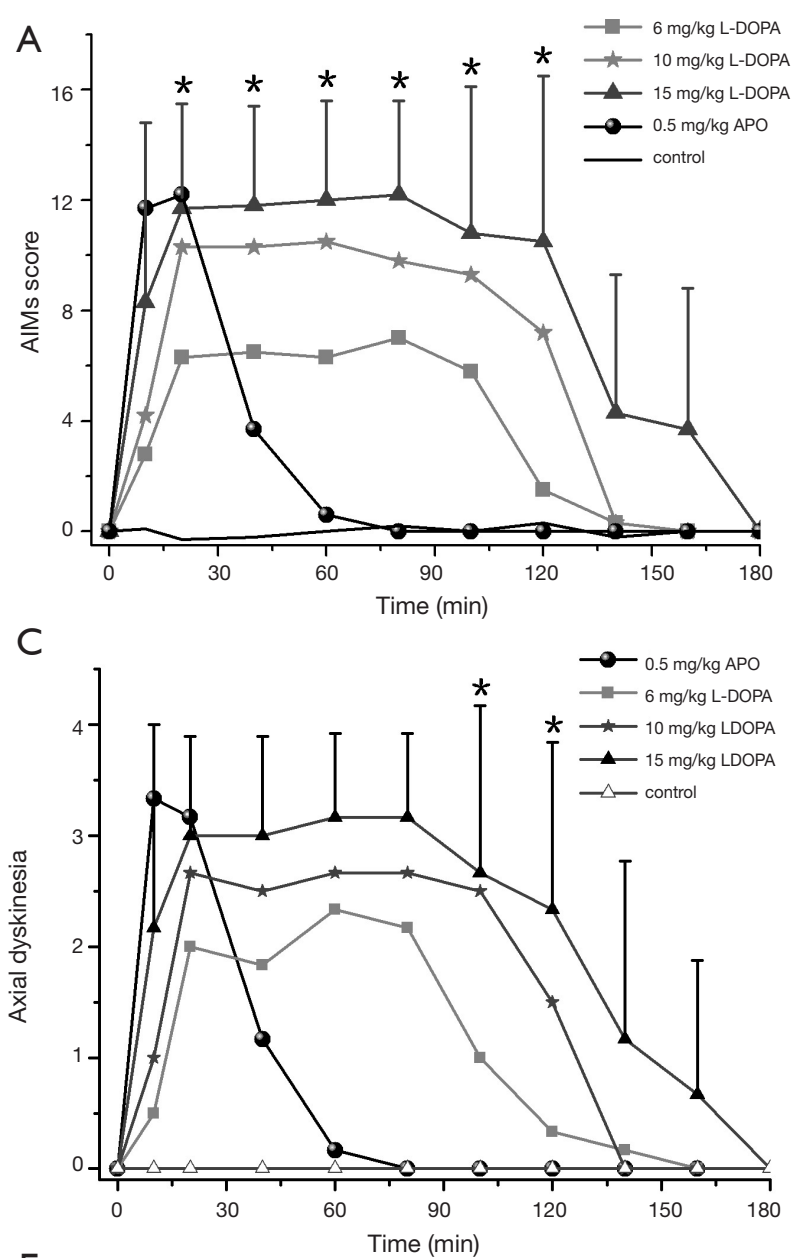

$\mathrm{E}$

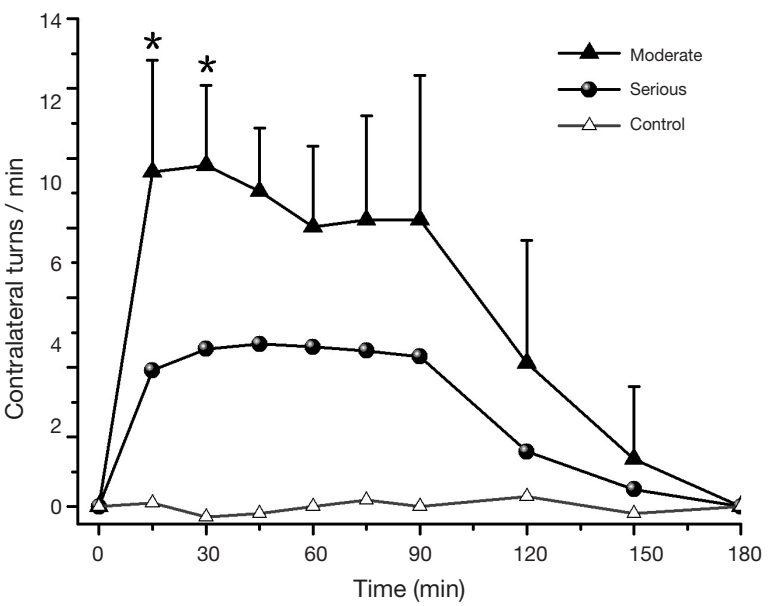

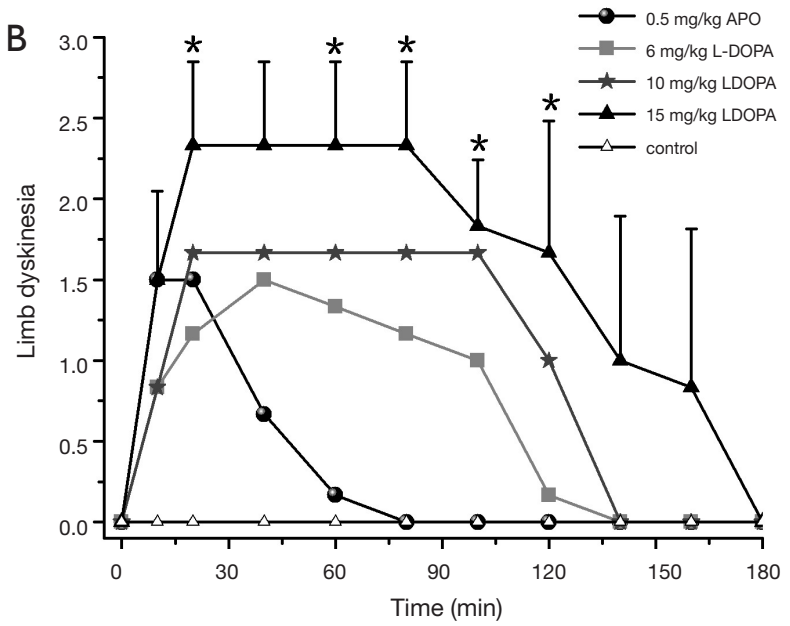

$\mathrm{D}$

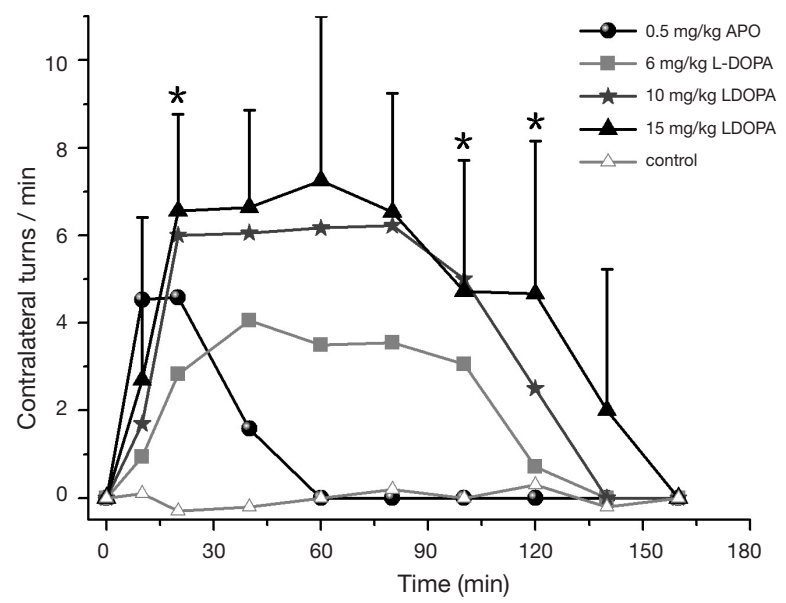

Figure 2 Dyskinesia and rotational movement were elicited by L-DOPA or APO. In (A,B,C,D), the rats of the serious lesion were injected $6,10,15 \mathrm{mg} / \mathrm{kg} \mathrm{L}$-DOPA and $0.5 \mathrm{mg} / \mathrm{kg}$ APO separately. In (A), the integrated scores of axials, limb, orolingual, and locomotive AIM were shown. In (B) the limb AIM, in (C) the axial AIM and in (D), the contralateral turns were shown. In (E), the contralateral turns in both serious and moderate lesion rats were showed after injecting $0.5 \mathrm{mg} / \mathrm{kg}$ APO. *, $\mathrm{P}<0.05,6 \mathrm{mg} / \mathrm{kg}$ L-DOPA $v s .15 \mathrm{mg} / \mathrm{kg}$ L-DOPA in (A,B,C,D); and in (E) serious lesion vs. moderate lesion. L-DOPA, levodopa; APO, apomorphine; AIM, abnormal involuntary movement. 

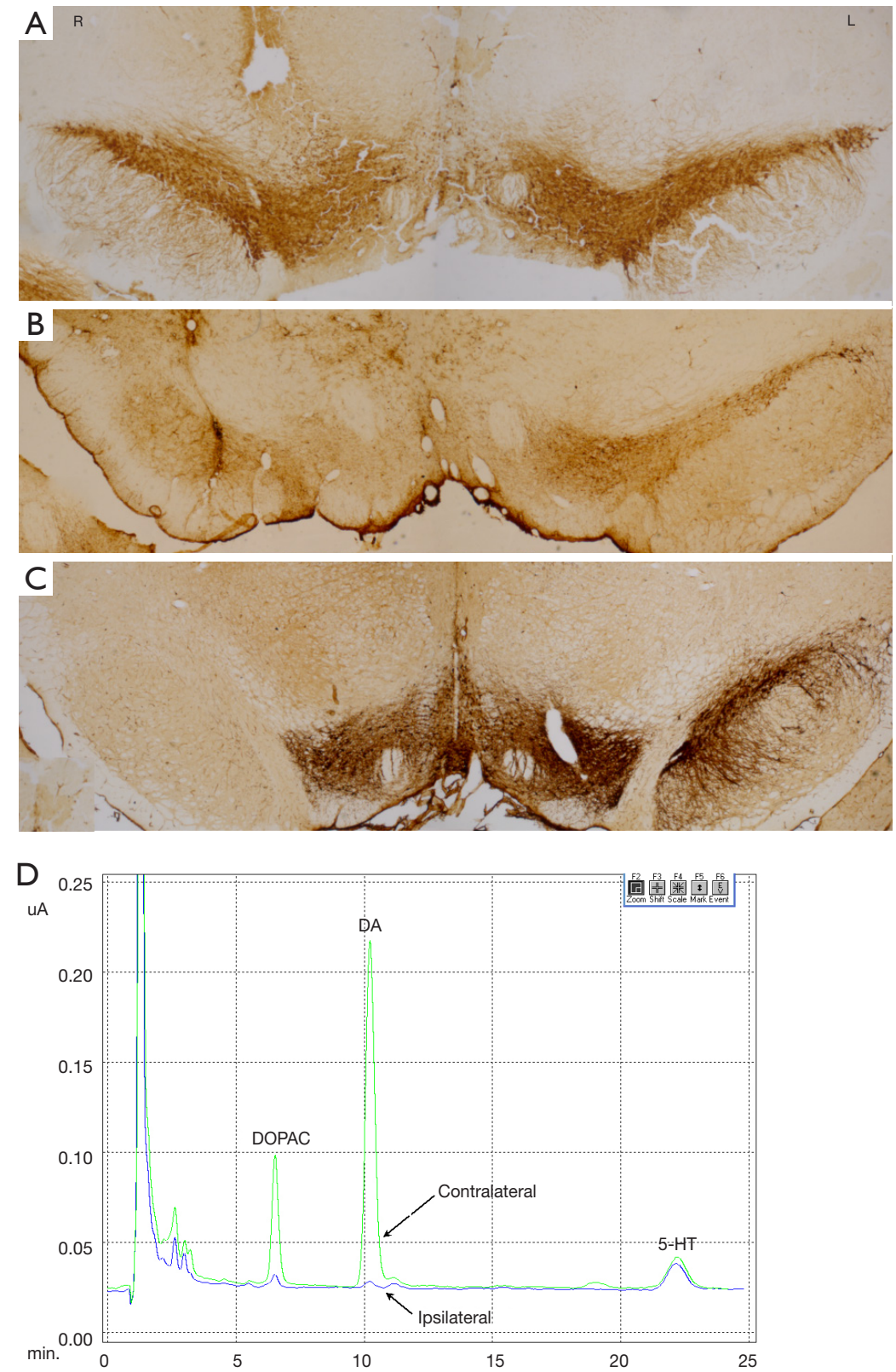

29-RIGHT 29-LEFT

Figure 3 Dopaminergic degeneration in SN and striatum induced by 6-OHDA. TH immunohistochemistry in SN of rat brain (A,B,C) shew that the rats of control (A) presented no dopaminergic neuron degeneration, and the moderate-lesion (B) and serious-lesion (C) rats presented different-degree degeneration of dopaminergic neurons. The right side $(\mathrm{R})$ is ipsilateral to the lesion side. Determination by HPLC shows the tissue level of DA reduced about $99 \%$ in the striatum ipsilateral to the lesion side compared with the contralateral intact side (D) in serious-lesion rats. The tissue levels of DOPAC and 5-HT were shown in the figure. SN, substantia nigra; 6-OHDA, 6-hydroxydopamine. 


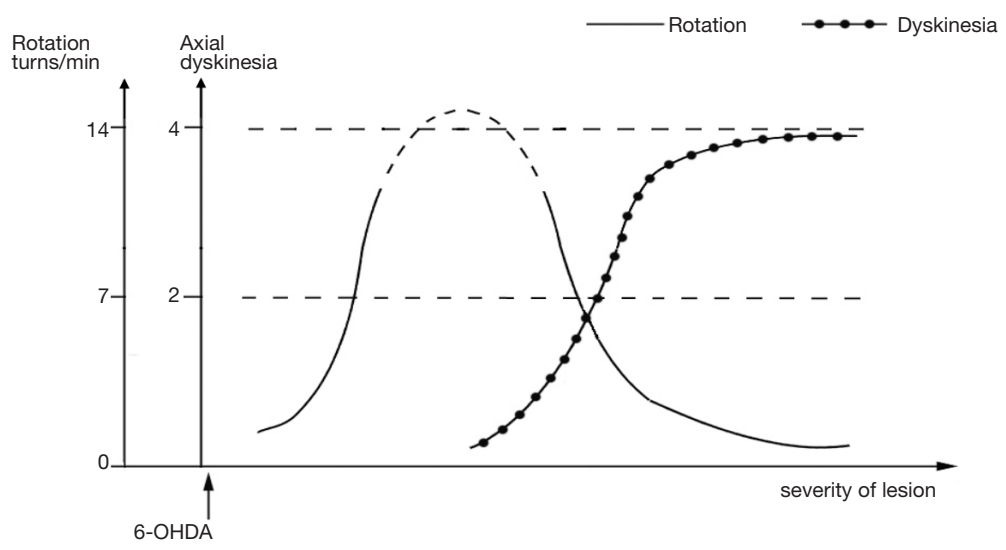

Figure 4 Behavioral characters elicited by L-DOPA or APO in the rats suffered lesions of varying severity induced by 6-OHDA. When the lesion is moderate, the greater the severity of lesion is, the faster the rotation elicited by L-DOPA or APO is, and there is no axial AIM. When the lesion becomes serious and reaches a certain degree, the axial AIM behavior will be elicited by L-DOPA or APO, and the greater the severity of lesion is, the slower the rotation is, and the more AIMs there will be. (hypothetic curve based on the literature and observations from the authors). L-DOPA, levodopa; APO, apomorphine; AIM, abnormal involuntary movement.

declines in the striatum of serious-lesion rats (Figure 3D). The behavioral testing shows that both the frequency of use of the forelimb contralateral to lesion side and the movement-coordination abilities decline significantly in moderately and seriously lesioned rats (Figure 1). So, we confirm that the rats indeed have suffered moderate and serious dopaminergic-degeneration induced by 6-OHDA.

When first elicited by L-DOPA or APO, the rats can present AIMs and/or rotational movements. The rats with moderate lesion by 6-OHDA $(20 \mu \mathrm{g})$ show no AIM except rotational movements, which are faster significantly than that of the rats with serious lesions after injecting APO (Figure $2 E, \mathrm{P}<0.05$ ). The rats with serious lesions by 6-OHDA (40 $\mu \mathrm{g})$ exhibit AIMs and rotational movements after first injecting L-DOPA or APO (Figure $2 A, B, C, D$ ). The AIMs include axial, limb, orolingual, and locomotive movements, extremely like the descriptions of dyskinesia in the literature (3-6).

\section{The relationships among lesions of varying severity elicited agent and AIMs}

The AIMs are compared to the elicited agents. In the present study, when elicited by APO, the duration of AIMs in both moderately and seriously lesioned rats is about 60-80 minutes. When elicited by L-DOPA of different concentrations, the duration is always about $150-180$ minutes in serious-lesion rats. So, the duration of AIMs may be related to the type of agent. On the other hand, when elicited by L-DOPA/APO, the larger the dosage of the agent is, the more severe the AIMs are (8), and the faster the rotation is (13). Therefore, the category and dosage of dopamine receptor agonists can affect the AIMs elicited by itself.

The AIMs are also compared to the degree of dopaminergic degeneration. The 6-OHDA-lesion rats can be regarded as successful PD model if its rotation speed is $\geq 210$ turns/30 $\mathrm{min}$ (7 turns/min) elicited by APO $(10,14)$, and the more severe the dopaminergic degeneration of rat models is, the faster the rotation is (13). The fastest rotation can reach 17 turns/min (8). However, when the lesion becomes serious and reaches a certain degree, the axial AIM will be elicited by L-DOPA or APO, and the greater the severity of lesion is, the slower the rotation is and the more AIMs there will be. In the present study, the rotation speed of moderate-lesion rats is 9 turns $/ \mathrm{min}$ (meet the criterion for PD models), but that of serious is only 4.5 turns/min elicited by APO. In the study of Lee et al. (4), the DA-denervated rats with severe AIMs (AIM score $>20)$ present lower controversies rotation than the rats with moderate AIMs (AIM score $\leq 20$ ) when elicited by L-DOPA. Therefore, there is no linear relationship 
between the rotational movement elicited by APO/L-DOPA to the whole degrees of dopaminergic degeneration.

Based on the literature and our observations, we speculate that the hypothetic relation-curve between dopaminergic lesion and AIMs elicited by L-DOPA or APO: when the lesion resulted from 6-OHDA is moderate, the greater the severity of lesion is, the faster the rotation elicited by L-DOPA or APO is, and there is no axial AIM; When the lesion becomes serious and reaches a certain degree, the axial AIM can be elicited by L-DOPA or APO, and the greater the severity of lesion is, the slower the rotation is and the more AIMs there will be (Figure 4). Because the axial AIM presents a better tendency among different degrees of damage in our research, as also exist in Winkler's study (8), the axial AIM was selected to represent AIMs in the hypothetic curve. Therefore, both dyskinesia and rotation are the behavioral expressions elicited by L-DOPA or APO in 6-OHDA lesion rats, and the rotation can also be regarded as an AIM in some sense. There is no absolute boundary between dyskinesia and rotation behavior, which reflect the damages of different severity in the nigrostriatal DA pathway, respectively.

There are several deficiencies in the present research, including the absence of tissue level of DA in moderatelesion rats and the fewer lesion gradings leading to inaccurate trend lines. Further studies deserve performing.

\section{Conclusions}

Both dyskinesia and rotation movement are the specific expressions elicited by L-DOPA or APO in rats with dopaminergic degeneration induced by 6-OHDA, which reflect the damages of different severity, respectively. There is no absolute boundary between dyskinesia and rotation movement. The dyskinesia will appear when the damage reaches a certain degree; since then, the greater the severity of dopaminergic degeneration is, the slower the rotation is, and the more AIMs there will be.

\section{Acknowledgments}

Funding: None.

\section{Footnote}

Conflicts of Interest: Both authors have completed the ICMJE uniform disclosure form (available at http://dx.doi. org/10.21037/apm.2020.03.32). The authors have no conflicts of interest to declare.

Ethical Statement: The authors are accountable for all aspects of the work in ensuring that questions related to the accuracy or integrity of any part of the work are appropriately investigated and resolved. The study was approved by the Ethical Committee of the Guangzhou Medical University (No. 2018-218).

Open Access Statement: This is an Open Access article distributed in accordance with the Creative Commons Attribution-NonCommercial-NoDerivs 4.0 International License (CC BY-NC-ND 4.0), which permits the noncommercial replication and distribution of the article with the strict proviso that no changes or edits are made and the original work is properly cited (including links to both the formal publication through the relevant DOI and the license). See: https://creativecommons.org/licenses/by-nc-nd/4.0/.

\section{References}

1. Paolini Paoletti F, Tambasco N, Parnetti L. Levodopa treatment in Parkinson's disease: earlier or later? Ann Transl Med 2019;7:S189.

2. Ahlskog JE, Muenter MD. Frequency of levodopa-related dyskinesias and motor fluctuations as estimated from the cumulative literature. Mov Disord 2001;16:448-58.

3. Padovan-Neto FE, Echeverry MB, Tumas V, et al. Nitric oxide synthase inhibition attenuates L-Dopa-induced dyskinesias in a rodent model of Parkinson's disease. Neuroscience 2009;159:927-35.

4. Lee CS, Cenci MA, Schulzer M, et al. Embryonic ventral mesencephalic grafts improve levodopa-induced dyskinesia in a rat model of Parkinson's disease. Brain 2000;123:1365-79.

5. Wu N, Wan Y, Song L, et al. The abnormal activation of D1R/Shp-2 complex involved in levodopa-induced dyskinesia in 6-hydroxydopamine-lesioned Parkinson's rats. Neuropsychiatr Dis Treat 2018;14:1779-86.

6. Wang XS, Zhang ZR, Zhang XR, et al. Modulation of CaMKIIa-GluN2B interaction in levodopa-induced dyskinesia in 6-OHDA-lesioned Parkinson's rats. Biomed Pharmacother 2018;107:769-76.

7. Cenci MA, Lundblad M. Post-versus presynaptic plasticity in L-DOPA-induced dyskinesia. J Neurochem 2006;99:381-92.

8. Winkler C, Kirik D, Björklund A, et al. L-DOPA-induced dyskinesia in the intrastriatal 6-hydroxydopamine model 
of parkinson's disease: relation to motor and cellular parameters of nigrostriatal function. Neurobiol Dis 2002;10:165-86.

9. Karhunena H, Virtanena T, Schallert T, et al. Forelimb use after focal cerebral ischemia in rats treated with an $\alpha 2$ -adrenoceptor antagonist. Pharmacology, Biochemistry and Behavior 2003;74:663-9.

10. Ungerstedt U. 6-Hydroxydopamine induced degeneration of central monoamine neurons. Eur J Pharmacol 1968;5:107-10.

11. Cenci MA, Whishaw IQ, Schallert T. Animal models of neuro-logical deficits: how relevant is the rat? Nat Rev

Cite this article as: Wang R, Shao M. L-DOPA-elicited abnormal involuntary movements in the rats damaged severely in substantia nigra by 6-hydroxydopamine. Ann Palliat Med 2020;9(3):947-956. doi: 10.21037/apm.2020.03.32
Neurosci 2002;3:574-9.

12. Carmen LS, Gage FH, Shults CW. Partial lesion of the substantia nigra: relation between extent of lesion and rotational behavior. Brain Res 1991;553:275-83.

13. Kirik D, Rosenblad C, Bjorklund A. Characterization of behavioral and neurodegenerative changes following partial lesions of the nigrostriatal dopamine system induced by intrastriatal 6-hydroxydopamine in the rat. Exp Neurol 1998;152:259-77.

14. Hefi F, Melamed E, Wurtman RJ. Partial lesions of the dopaminergic nigrostriatal system in rat brain: biochemical characterization. Brain Res 1980;195:123-37. 